\title{
Nanocatalysis: hypothesis on the action mechanism of gold
}

\author{
B. L. Oksengendler ${ }^{1}$, B. Askarov ${ }^{1}$, I. N. Nurgaliyev ${ }^{1}$, S. E. Maksimov², V. N. Nikiforov ${ }^{3}$ \\ ${ }^{1}$ Institute of chemistry and physics of polymers,Uzbek Academy of Sciences, \\ Tashkent, Uzbekistan \\ ${ }^{2}$ Institute of ion-plasma and laser technologies,Uzbek Academy of Sciences, \\ Tashkent, Uzbekistan \\ ${ }^{3}$ Physics Department, M.V.Lomonosov State University, Moscow, Russia \\ oksengendlerbl@yandex.ru,dr.asqarov@mail.ru,ilnar82@mail.ru, \\ maksimov_s@yahoo.com,pppnvn@yandex.ru
}

\section{PACS 75.50.Tt, 81.16.Hc, 87.85.Rs $\quad$ DOI 10.17586/2220-8054-2015-6-2-249-261}

In this article, the problem of nanocatalysis is considered when the catalysts are gold nanoparticles. The main experimental facts are presented and basic qualitative dependences are highlighted. The hypothesis considers the role of Tamm states of gold nanoparticles, with the modification of these states to reduce nanoparticle sizes. A semi-quantitative quantum-chemical reaction scheme of oxygen dissociation with gold nanocatalysis is shown. A theoretical answer to the basic experimental test has been obtained.

Keywords: nanocatalysis, d-atoms, Tamm levels.

Received: 2 February 2015

\section{Introduction}

Although the catalytic properties of $\mathrm{Au}$ (hydrogen-deuterium exchange, the reduction of $\mathrm{NO}_{\mathrm{x}}$ by using of $\mathrm{H}_{2}$, isomerization of paraffins, partial oxidation) had been quite widely studied until the end of the 1980's [1-6], it was found that Au is a much less efficient catalyst than the group VIII elements of Mendeleev's table and other transition metals. However, in 1987, it was found by Haruta et al. [7-9] that small size Au particles increased the activity of CO oxidation dramatically, performing the reaction at temperatures below those of such classical catalysts as Pt [7-10]. This circumstance greatly increased the interest in gold as a catalyst [11-18].

The main results of the basic experiment are the following:

1) The growth of the catalytic activity was observed for nanoparticles with size less than $\mathrm{R}=50 \AA$, particularly for nanoparticles with $\mathrm{R}<30 \AA$ [8-10; 19-29].

2) The increased catalytic activity was observed as in small Au particles [23], in complexes of the partially oxidized/metal particles [30,31], in gold atom clusters $[32,33]$ and in the cationic $\mathrm{Au}$ particles.

3) The role of the substrate in the catalysis of CO oxidation by Au particles was also important $[8-11,30]$.

4) Most catalytic activity was observed for $\mathrm{Au}$ nanoparticles on $\mathrm{TiO}_{2}$ and $\mathrm{Fe}_{2} \mathrm{O}_{3}$ substrates [8-11, 24].

5) It was assumed that the activation of $\mathrm{O}_{2}$ molecule took place at the metal-substrate interface [36, 37], or directly on the substrates [8-11,19,21]. 
6) Changes in some properties of Au clusters are thought to vary as the function of their sizes; which are assumed to be important in the nanocatalysis problem:

a) the structural phase transition from FCC (bulk material) to the dodecahedral cluster (at $R=25 \AA$ ) and icosahedral cluster (at $R=16 \AA$ ) [38] is discussed; there are data that the latter transition is observed at a much larger size (not at 100 atoms, but at 550 atoms in the cluster) [39]. Note that the transition to the icosahedron was experimentally observed in Y-zeolites at $\mathrm{R}=10 \AA$ [41] and in 50-atomic Pt clusters on carbon [40];

b) there was a decrease in the bond lengths of $\mathrm{Cr}, \mathrm{Fe}, \mathrm{Cu}, \mathrm{Ag}, \mathrm{Pd}, \mathrm{Au}, \mathrm{Pt}$ metals at the size $\mathrm{R}<30 \AA$ [42-53]; so, at $\mathrm{R}=30 \AA$ the length of $\mathrm{Au}-\mathrm{Au}$ bond is $\mathrm{d}=2.84 \AA$, and at $\mathrm{R}=8 \AA$ it is $\mathrm{d}=2.72 \AA$, whereas the bulk material has $\mathrm{d}=2.88 \AA[48,49,51]$.

c) such shortening of the bond lengths is characteristic for metal dimers (Fe, Mg) [54]; reduction of the $\mathrm{Au}-\mathrm{Au}$ bond length also occurs in clusters;

d) there was a reduction of the bond lengths in the metal particles on the substrates, like carbon, which are weakly interacting with the metal particles $[52,55,56]$;

e) there are changes in other properties of metal clusters (electronic, magnetic, chemical) at the scale of few nanometers [57, 64]; the most important of which is the modification of the electronic spectrum, namely, the displacement of d-band of $\mathrm{Cu}$ in its atomic spectrum with decreasing particle size[60]; the same effect was also observed for Pt and Pd [59].

The appearance of a gap in the spectrum at the Fermi level $\left(\mathrm{E}_{e}=0.45 \mathrm{eV}\right)$ is observed for $\mathrm{Au}$ clusters with $\mathrm{R}<19 \AA$ on a carbon substrate, so that there is a metal-insulator transition; a similar effect is also observed for two-dimensional particles of $\mathrm{Au}$ (200 atoms) on a $\mathrm{TiO}_{2}$ substrate with $\mathrm{E}_{e}=0.2-0.6 \mathrm{eV}$ [23] (Figure 1).

Concerning the theoretical interpretation of the Au nanoparticles' unique catalytic activity, only general considerations have been expressed:

a) about the role of reducing the coordination number of surface atoms;

b) about the general increase of the relative part of the total number of surface atoms;

c) about the existence of the evident correlation between the increased catalytic activity and the existence of metal-insulator transition with decreased cluster size;

d) about the correlation of the increase in nanoparticle catalytic activity and the decrease of the interatomic $\mathrm{Au}-\mathrm{Au}$ distance in smaller cluster sizes;

e) about the role the substrate plays in increased catalytic activity.

The most important of these five positions are the positions c) and e), i.e. that the high catalytic activity of the gold nanoparticles promotes the formation of a gap in the electronic spectra of nanoparticles. In this regard, it is very important to discuss of the role of Tamm states in the nanoparticle, taking into account their modification with decreased nanoparticle size.

\section{Tamm states of nanoparticles}

In 1932, on the basis of quantum mechanics, I.E.Tamm for the first time demonstrated that apart from the band states of electrons in a crystal known at that time, electron states of a completely different type can exist on a crystal surface [65]. These surface electron states have a discrete energy spectrum and wavefunctions that exponentially decay with increasing distance from the surface to within or away from the crystal. During more than 80 years since then, the theory of surface states has been considerably developed with regards to computational techniques and the analysis of such states in various crystals $[66,67]$. Due to the evolution of nanoscience, the problem of surface states has become even more relevant. However, general analysis of the surface states problem shows that basically new factors that were not reflected in the proposed model should be considered when applied 

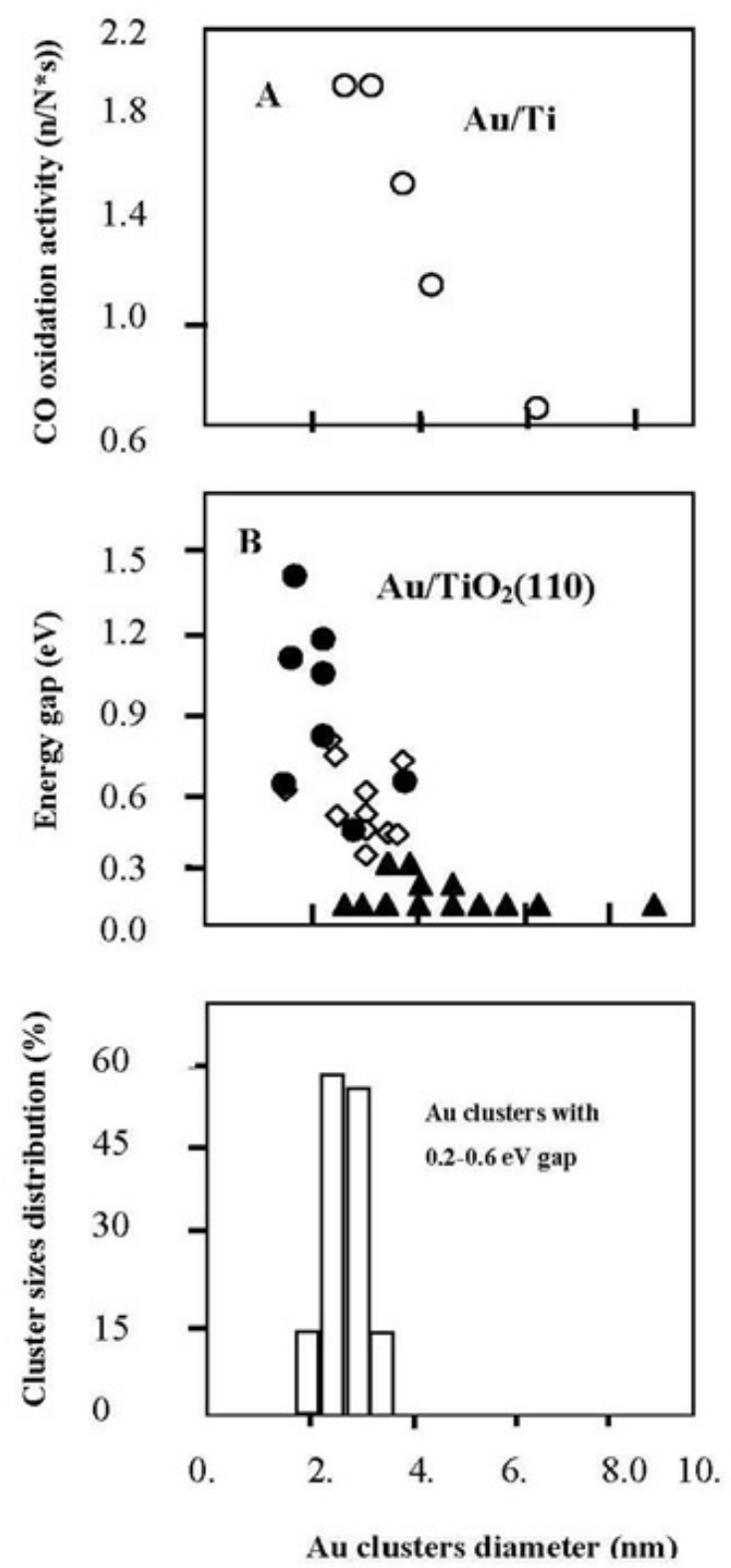

FIG. 1. Dependence of the CO oxidation activity, the energy gap in the electronic spectrum of the gold nanoparticles and their size distribution on $\mathrm{Au}$ cluster size

to nanoclusters. Concepts such as the role of the surface curvature (nonplanar surface), the sharpness of the potential barrier simulating the width of the surface region (diffuse nature of the interface), and finiteness of the nanocluster size (in contrast to the case of a semi-infinite crystal) should be introduced into the theory. At the same time, nanoparticles have a characteristic that describes the above properties in an integrated manner; we are speaking of confinement of elementary excitations in a nanoparticle. The most important property determined by the confinement of electrons is the discretization of their electronic spectra, which accompanies the increase in the spectral gap width; the broadening of the gap increases when the characteristic size of a nanoparticle decreases [4]. Therefore, it is reasonable to consider the features introduced into the Tamm surface states of nanoparticles by a fundamental property like the electron confinement. 


\section{1. $\quad$ Model}

Our analysis is based on a combination of three models: the Tamm model of surface states [65], the well-known Kronig-Penney model of a solid [68], and results obtained by Efros on the electron structure of a nanosphere [69]. For this purpose, each model will be slightly developed.

The basic scheme of Tamm's analysis [65] is shown in Fig. 2.

bxa

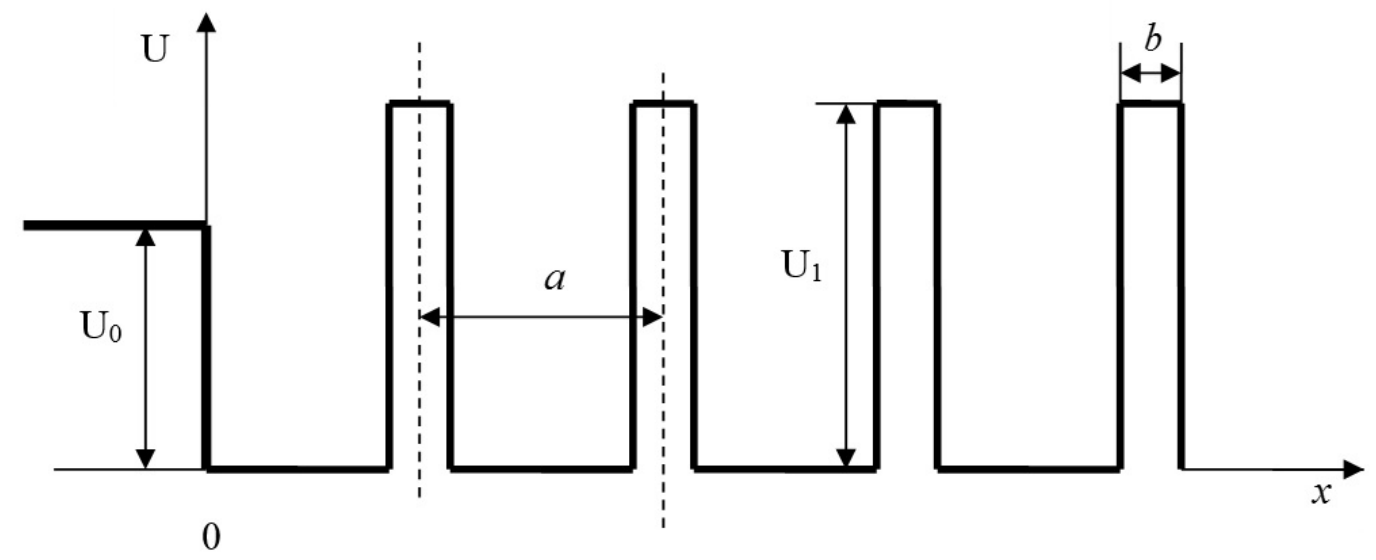

FIG. 2. Potential energy of a crystal with a surface in the Tamm- Kronig-

Penney model

Tamm presented the wavefunction of a surface electron state outside the crystal (for $x<0)$ in the form:

$$
\Psi=A \exp \left[\frac{\sqrt{2 m\left(U_{0}-E\right)}}{h^{2}} x\right]
$$

where $E$ (energy of Tamm's state) satisfies the condition $0<E<U_{0}$.

Introducing the parameters (see Fig.2.):

$$
\xi=\frac{a}{h} \sqrt{2 m E} ; \quad q=\frac{a}{h} \sqrt{2 m U_{0}} ; \quad p=a b \frac{m U_{1}}{h^{2}},
$$

Tamm obtained the following equation for energy $E$ :

$$
\xi \operatorname{ctg} \xi=\frac{q^{2}}{2 p^{2}}-q \sqrt{1-\frac{\xi^{2}}{q^{2}}} .
$$

Let us simplify this expression for low energies $(\xi<<1)$ :

$$
E\left(q a^{2}-\frac{2 m}{h^{2}}\right)=q-\frac{q^{2}}{2 p^{2}}
$$

Thus, we obtain the dependence of energy $E$ on the dimensionless parameter $p$, appearing in the Kronig-Penney model [69]. This model can also be represented in Fig.2 if we consider the range of $X>>0$. The equation for allowed energy values in the Kronig-Penney model has the form (in notation used in [69-70]):

$$
\left\{\begin{array}{l}
\left|\cos \left(k a-\operatorname{actg}\left(\frac{\Omega a}{k a}\right)\right)\right| \leqslant \frac{1}{\sqrt{1+\left(\frac{\Omega a}{k a}\right)^{2}}} \\
E=\frac{h^{2}}{2 m a^{2}}(k a)^{2}
\end{array}\right\},
$$


where $\Omega a$ characterizes the dimensionless barrier penetrability. For low energies, $(\Omega a / k a>>1)$, Eq.(5) is transformed to:

$$
|\sin (k a)| \leqslant \frac{k a}{\Omega a} .
$$

Expanding $\sin (k a)$ (for $k a<1$ ), we obtain the first two solutions $E=f(\Omega a)$ :

$$
E_{1}=0 ; \quad E_{2}=\frac{h^{2}}{2 m a^{2}} 6\left(1-\frac{1}{\Omega a}\right)^{2} .
$$

The graphical form of Eq. (6) is shown in Fig.3.

Combining Fig. 3 with Eq. (7), we obtain penetrability parameter $\Omega a$ as a function of the first band gap:

$$
\begin{gathered}
E_{\text {gap }}^{1}=E_{2}-E_{1}: \\
\frac{1}{\Omega a}=1-\sqrt{\frac{E_{\text {gap }}^{1}}{h^{2} / 2 m a^{2}}} / 6 .
\end{gathered}
$$

Matching the Kronig-Penney model and the Tamm model by the condition $p=\Omega a$, we obtain from expressions (8) and (4):

$$
\frac{E}{h^{2} / 2 m a^{2}}=2 \sqrt{\frac{U_{0}}{h^{2} / 2 m a^{2}}}-\frac{U_{0}}{h^{2} / 2 m a^{2}}-\left[1-\frac{1}{6} \sqrt{\frac{E_{g a p}^{1}}{h^{2} / 2 m a^{2}}}\right]^{2} .
$$

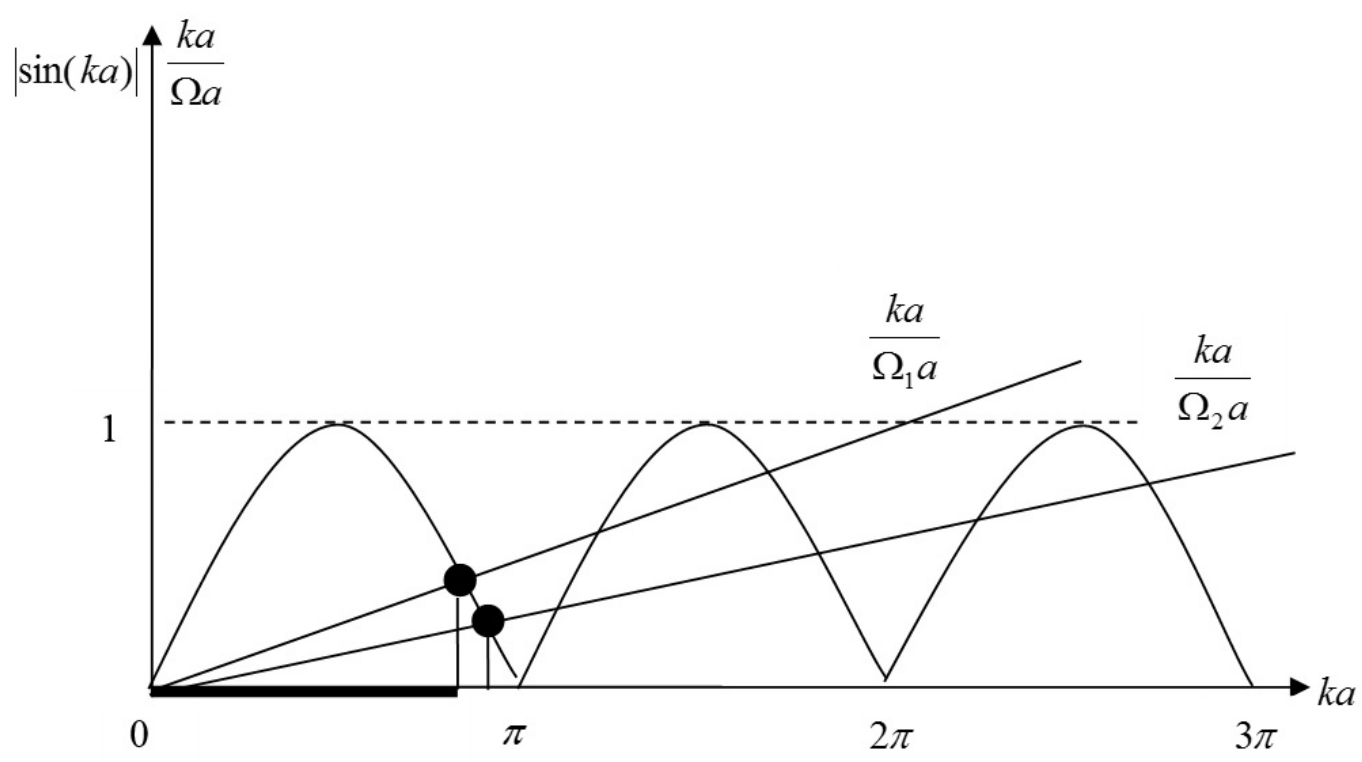

FIG. 3. Plot for determining the boundaries of allowed and forbidden bands (bullets); $\Omega_{1}<\Omega_{2}$

Let us now consider the dependence of gap width $E_{\text {gap }}^{1}$ on radius $(R)$ of a nanosphere [68]:

$$
E_{\text {gap }}^{1}=E_{g}^{0}+\frac{A}{R^{2}}, A \equiv h^{2} \pi^{2} / 2 m
$$

where $E_{g}^{0}$ is the gap width for the bulk material. Substituting expressions (10) into (9), we obtain the following dependence of the position of the Tamm level on nanoparticle radius $R$ :

$$
E=\frac{h^{2}}{2 m a^{2}} \varphi(R)
$$


where:

$$
\varphi(R) \equiv\left\{2 \sqrt{\frac{U_{0}}{h^{2} / 2 m a^{2}}}-\frac{U_{0}}{h^{2} / 2 m a^{2}}-\left[1-\frac{1}{6} \sqrt{\frac{E_{g}^{0}+A / R^{2}}{h^{2} / 2 m a^{2}}}\right]\right\} .
$$

It should be noted that $d \varphi(R) / d R<0$.

Thus, exponent $\alpha$ in the wavefunction $\psi(x)=A \exp (\alpha x) ;(x<0)$ for the Tamm electron has the form:

$$
\alpha=\frac{1}{a} \sqrt{\frac{U_{0}}{h^{2} / 2 m a^{2}}-\varphi(R)} .
$$

Analyzing Eq. (13), we can easily see that the value of $\alpha$ decreases with $R$ :

$$
\frac{d \alpha}{d R}>0
$$

Consequently, we obtain two important results (Fig.4):

1) upon a decrease in nanoparticle radius $R$, the Tamm level increases, approaching $U_{0}$ from below;

radius $R$.

2) the degree of localization of Tamm wavefunction $\alpha$ decreases upon a decrease in

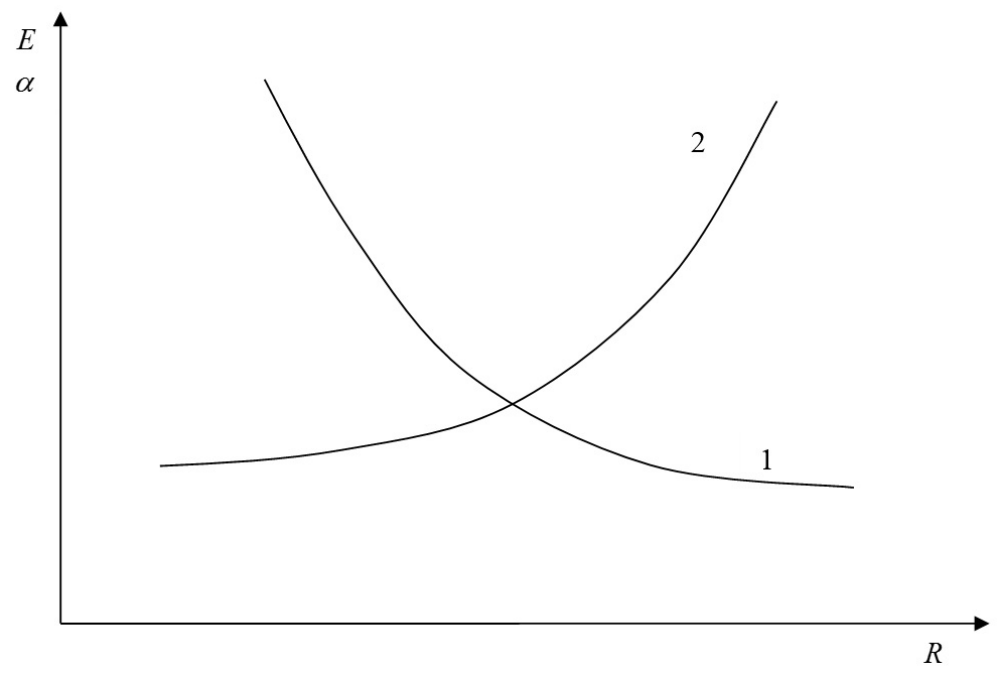

FIG. 4. Schematic qualitative dependence of the energy of Tamm's level (1) and damping factor of the wavefunction of a Tamm electron (2) on the nanoparticle radius

\section{Model of nanocatalysis of the oxygen molecule dissociation by Au nanoclusters}

The geometric model diagram for the chemical interaction of an oxygen molecule with a nanocatalytic gold cluster is shown in Fig. 5.

It is clear that the Tamm orbital of gold cluster increasingly penetrates into the space between the oxygen atoms as the oxygen molecule approaches the nanocluster; we also note that since the empty state of the oxygen molecule $\sigma_{p_{z} S}^{*}$ is antibonding, there is a lack of electron density at the center of the molecule. Furthermore, it can be assumed that the appearance of electron density of the Tamm states in the center of the oxygen molecule will lead to Coulomb repulsion of the two oxygen atoms, so that the interaction of the molecule 


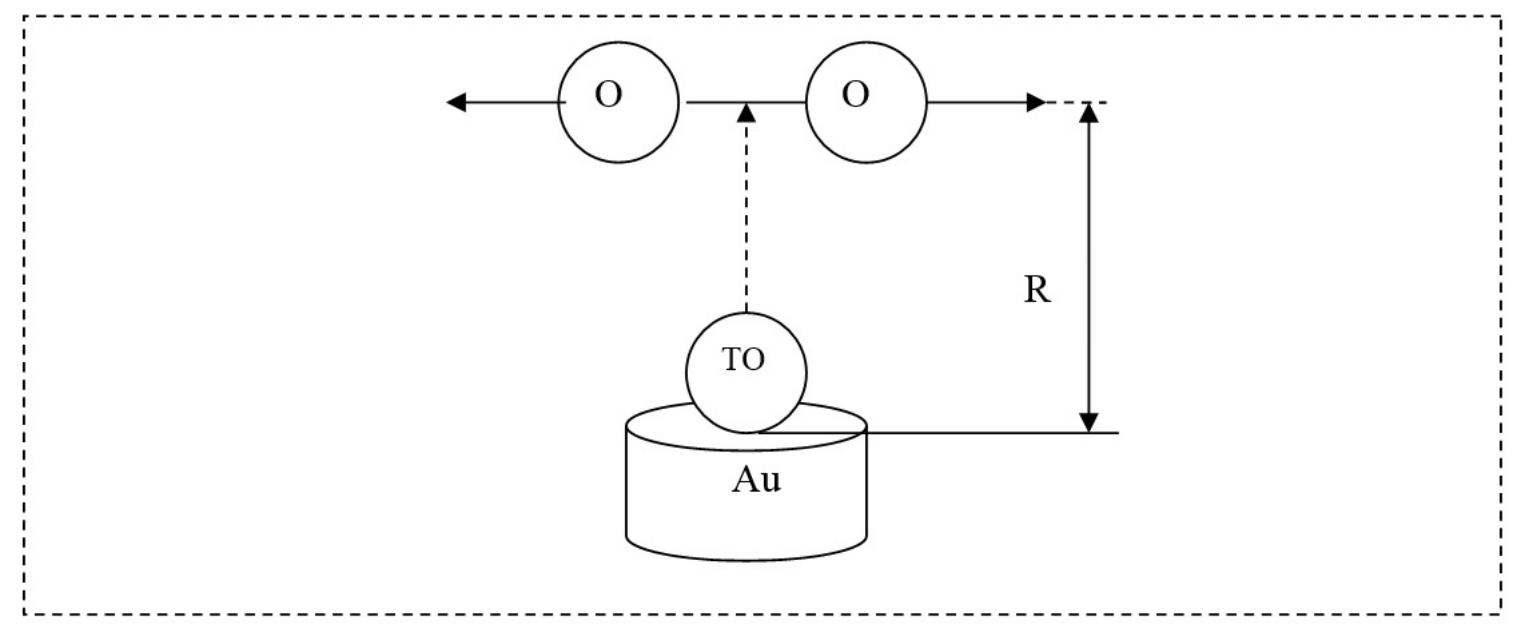

FIG. 5. Geometric scheme for oxygen molecule interaction with Tamm state of gold nanocluster

with the nanocluster occurs through the synchronous reaction: approach of $\mathrm{O}_{2}$ with the nanocluster is accompanied by an increase in the distance between the oxygen atoms. From another point of view, separation of the oxygen atoms, according to the quantum chemistry laws [71], reduces the splitting of all levels (the drop of the exchange integral), so that the $\sigma_{p_{z} S}^{*}$ level is reduced (Figure 6).

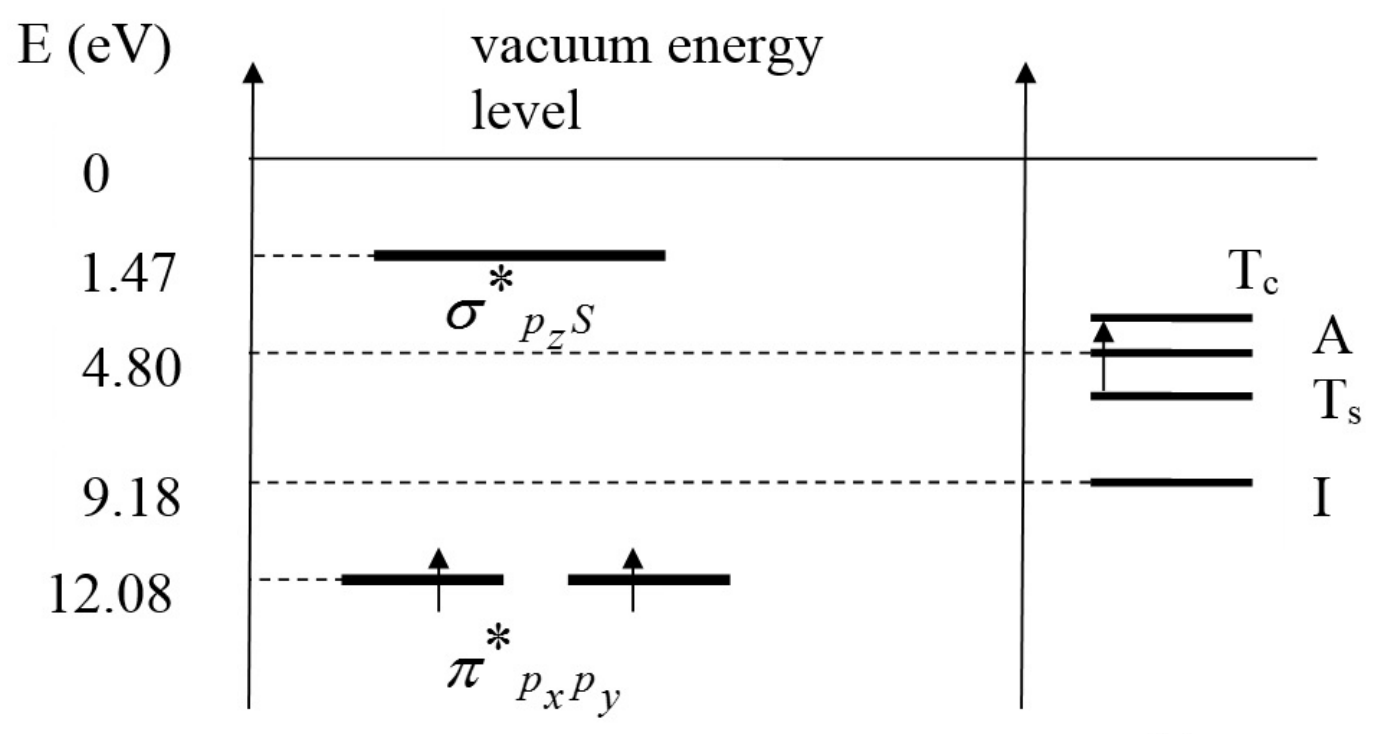

a)

b)

FIG. 6. Quantum-chemical model for the interaction of the electronic states of the oxygen molecule with Tamm states of gold nanoclusters; electronic energy levels of: a) the HOMO and LUMO of $\mathrm{O}_{2} ; \mathrm{b}$ ) I is the ionization potential of $\mathrm{Au}$ atom, $\mathrm{T}_{c}$ is the Tamm level of the Au nanocatalysts, $\mathrm{T}_{s}$ is the Tamm level of the semi-infinite Au catalyst, A is the work function of gold

Note further that Figure 6 shows the diagram of the electronic levels of the oxygen molecule and of the gold nanoclusters. We see that the Tamm state lies below the $\sigma_{p_{z} S}^{*}$ level, 
so lowering the $\sigma_{p_{z} S}^{*}$ level and its approach to the Tamm level promotes the superposition of the wave function for the free oxygen molecule and the wavefunction of the Tamm state. The total wavefunction of the "oxygen molecule + gold nanocluster" system has the form:

$$
\Psi(R)=C_{1}(R) \sigma_{p_{z} S}^{*}+C_{2}(R) \varphi_{T O}
$$

In this expression, $C_{1}(R)$ and $C_{2}(R)$ are the contributions of the $\sigma_{p_{z} S}^{*}$ oxygen states and of the Tamm orbitals $\varphi_{T O}$ in the total wave function of the combined system. Note that the coincidence of the lowering $\sigma_{p_{z} S}^{*}$ energy levels and of the Tamm states' flow of electrons into the oxygen molecule occurs most effectively, since the Franck-Condon factor is maximal in such a resonance [72]. From the point of view of quantum chemistry, the filling of the antibonding state of the oxygen molecule destabilizes it, i.e. reduces the dissociation energy of about $100 \mathrm{~kJ} / \mathrm{mol}$ [73]. Taking into account that the Tamm level may be filled by two electrons initially and they (couple) can overflow to the $\sigma_{p_{z} S}^{*}$ level, then energy reduction to zero takes place, so that the oxygen molecule dissociates spontaneously.

In this scheme, the question about the role of the size of the gold nanoclusters is of great interest. As was shown in the previous section, the nanocluster size affects both the length of the Tamm states' wave function and the depth of its position (Eqs. (11), (13)), so for smaller nanocluster, first, the Tamm wave function penetrates into the interoxygen space with the approach of the reagents earlier, and, second, the resonance of $\sigma_{p_{z} S}^{*}$ and $\varphi_{T O}$ also occurs earlier than in case of large clusters. Therefore, the effectiveness of the nanocluster catalyst in our model increases with decreasing gold nanocluster size, which is in full agreement with experimental results (see Fig. 1).

\section{Test of the model correctness}

It is desirable for the qualitative description of the gold nanoparticle catalysis model to be supported by quantitative confirmation. The criterion for the correctness of this theory can be the test of the dependence "catalysis efficiency" $\sim 1 / d^{3}$ ", where the $\mathrm{d}$ is the size of the gold nanoparticles (Figs. 1, 7) [14,74].

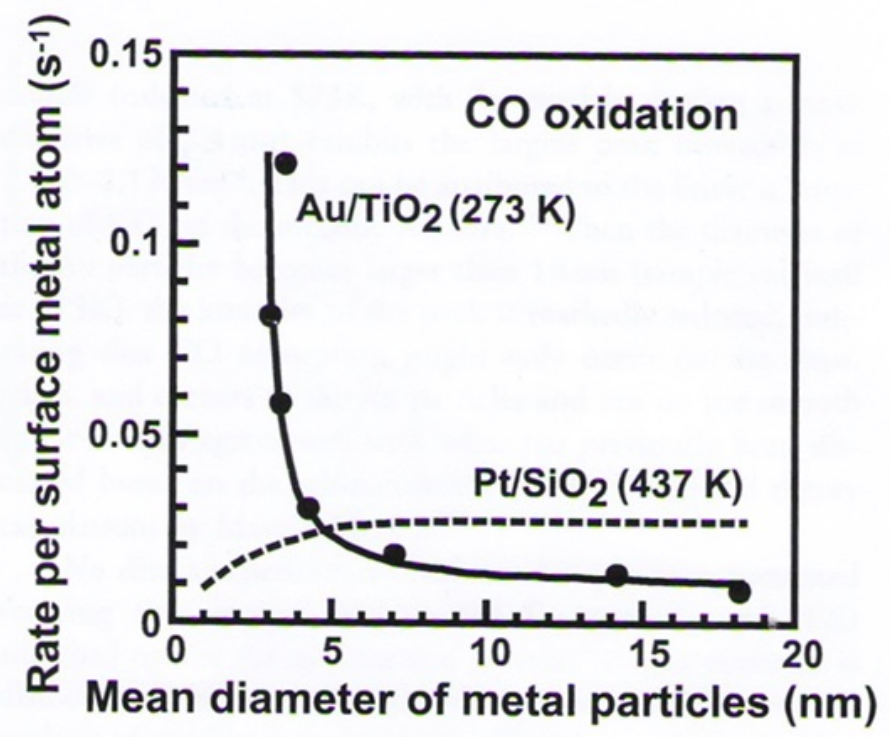

FIG. 7. Turnover frequency (TOF) for $\mathrm{CO}$ oxidation over $\mathrm{Au} / \mathrm{TiO}_{2}$ as a function of the mean diameter of $\mathrm{Au}$ particles [14,74] 
Note that the law " $1 / \mathrm{d}^{3}$ " is confirmed in a very broad class of experiments for both different substrates and types of contacts for Au nanoparticles with the substrates [74,75].

We consider such an approach of an $\mathrm{O}_{2}$ molecule to a $\mathrm{Au}$ nanoparticle, when the Tamm electron is already located between two oxygen atoms (Fig. 8).

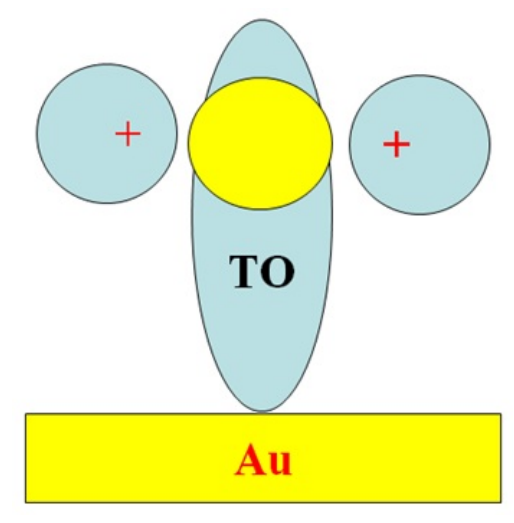

Fig. 8. Hellmann-Feynman scheme for the change of the dissociation energy of oxygen molecule at the penetration of Tamm orbital into the center of the molecule

According to the Hellmann-Feynman theorem [70], the resulting system can be considered electrostatically, so that the activation energy $\mathrm{Q}_{0}$ of dissociation process of the oxygen molecule decreases by $\Delta \mathrm{Q}$, which is proportional to the part of the Tamm state electron density located between the two oxygen atoms (shaded part of orbital in Fig.8), i.e.:

$$
\Delta \mathrm{Q}=\beta \frac{1}{\alpha}\left(\frac{C_{2}}{C_{1}}\right)^{2}
$$

where $\beta$ is the proportionality coefficient, $\frac{1}{\alpha}$ is the "size" of Tamm wave function (see (15)), $\left(\frac{C_{2}}{C_{1}}\right)^{2}$ is the proportion of Tamm state penetrated into the space between the oxygen atoms. According to the two-center chemical bond theory [70], we have:

$$
\left(\frac{C_{2}}{C_{1}}\right)^{2}=\frac{1}{4}\left(l-\sqrt{l^{2}+4}\right)^{2},
$$

where $l=\frac{H_{11}-\left(H_{22}-E\right)}{H_{12}}, H_{12}$ is the exchange integral of the states of $\left(\sigma_{P_{Z} S}^{*}\right)$ antibonding of the oxygen molecule and of the Tamm state (see. (15)); $-H_{11}=J_{1}$ is the ionization potential of $\sigma_{P_{Z} S}^{*}$ antibonding; $-H_{22}^{0}=J_{2}$ is the ionization energy of the Tamm state of semi-infinite $\mathrm{Au}$ crystal, $\mathrm{E}$ is the energy of the Tamm state of $\mathrm{Au}$ nanoparticles.

It is essential that the value $\frac{1}{\alpha}\left(\frac{C_{2}}{C_{1}}\right)^{2}$ depends on the function $\varphi(R)$ ( $\alpha$ is based on Eq.(13), $\left(\frac{C_{2}}{C_{1}}\right)^{2}$ is based through E on Eq. (11)), and therefore from the fact that $\varphi(R)$ has a small value, it is easy to obtain:

$$
\frac{1}{\alpha}\left(\frac{C_{2}}{C_{1}}\right)^{2}=\frac{a}{\sqrt{M-\varphi(R)}} \frac{L}{2}[\tilde{L}+\varphi(R)],
$$

where the dimensionless quantities $L, \tilde{L}$ and $M$ are the functions of $\hbar^{2} / 2 m a^{2}, U_{0}, E_{g}^{0}, H_{11}$ and $\mathrm{H}_{12}$. 
Further, freed from the radicals in the $\varphi(R)$ function, we easily obtain:

$$
\frac{1}{\alpha}\left(\frac{C_{2}}{C_{1}}\right)^{2} \approx B+C \frac{1}{R^{2}}+D \frac{1}{R^{4}}
$$

Now, we can write the expression for the probability of oxygen molecule dissociation on the surface of a Au nanoparticle:

$$
e^{-\frac{Q_{0}-\Delta Q}{k T}} \geqslant e^{-Q_{0} / k T}+e^{-Q_{0} / k T} \frac{Q_{0}}{k T} \beta \frac{1}{\alpha}\left(\frac{C_{2}}{C_{1}}\right)^{2} .
$$

Using (19), we obtain:

$$
e^{-\frac{Q_{0}-\Delta Q}{k T}} \geqslant e^{-Q_{0} / k T}+e^{-Q_{0} / k T} \beta\left[B+\frac{C}{R^{2}}+\frac{D}{R^{4}}\right] \frac{Q_{0}}{k T},
$$

where $B, C, D$ are the constants.

It is obvious that the bracketed expression is very close to the function Const $/ R^{3}$; in our view, this indicates reasonable agreement between the model and experiment (Fig. 7), which has not been obtained in other approaches [74, 75].

\section{Conclusion}

On the basis of the analysis of existing experimental data, it is found with a high degree of reliability that the catalytic activity of $\mathrm{Au}$ nanoparticles depends on a combination of three factors:

-size of the gold nanoparticles;

- type of substrate upon which the Au nanoparticle is placed;

- type of the contact of Au nanoparticles with the substrate.

The most important of these three factors is the first one, and it was found that a gap appears in the electronic spectrum of Au nanoparticles when the size of those nanoparticles is of $50 \AA$ or less, and that from this point, their catalytic properties sharply increase.

We have assumed that the catalytic activity of $\mathrm{Au}$ nanoparticles is related to features of the surface Tamm states formed in the gap of Au nanoparticle electronic spectra.

The above model of Tamm states in the nanoparticle showed that the properties of these states are modified with the decreasing nanoparticle size: Tamm orbitals "stretch" radially from the surface, and the energy levels of these states are raised. Using the quantumchemical model for $\mathrm{O}_{2}$ molecule dissociation on the surface of a $\mathrm{Au}$ nanoparticle showed that the catalytic activity of $\mathrm{Au}$ nanoparticles increase as their radii decrease as $\frac{\text { Const }_{1}}{R^{2}}+\frac{\text { Const }_{2}}{R^{4}}$, which is very close to the experimentally observed dependence " $1 / R$ " and can thus serve as a "litmus test" for the model, confirming its reliability.

\section{References}

[1] Bond G.C. The catalytic properties of gold. Gold Bull., 5(1), P. 11-13 (1972).

[2] Schwank J. Catalytic gold. Gold Bull., 16(4), P. 103-110 (1983).

[3] Hutchings G.J. Catalysis. A golden future. Gold Bull., 29(4), P. 123-130 (1996).

[4] Somorjai G.A., Park J.Y. Evolution of the surface science of catalysis from single crystals to metal nanoparticles under pressure. J. Chem. Phys., 128(18), P. 182504 (2008).

[5] Thompson D. New advances in gold catalysis. Part I. Gold Bull, 31(4), P. 111-118 (1998).

[6] Bond G.C., Thompson D.T. Catalysis by gold. Catal. Rev.Sci. Eng., 41(3-4), P. 319-388 (1999).

[7] Haruta M., Kobayashi T., Sano H., Yamada N. Novel Gold Catalysts for the Oxidation of Carbon Monoxide at a Temperature far Below $0^{\circ}$. Chem. Lett., 2, P. 405-408 (1987).

[8] Haruta M. Size and support dependency in the catalysis of gold. Catal. Today, 36, P. 153-166 (1997).

[9] Haruta M. Novel catalysis of gold deposited on metal oxides. Catal. Surv. Asia., 1, P. 61-73 (1997). 
[10] Haruta M. Gold as a low temperature catalyst: factors controlling activity and selectivity. In book: 3-World Congress on Oxidation Catalysis. Elsevier Science, Amsterdam, P. 123-134 (1997).

[11] Haruta M., Daté M. Advances in the catalysis of Au nanoparticles. Appl. Catal., A 222, P. 427-437 (2001).

[12] Grisel R.J.H., Weststrate K.-J., Gluhoi A., Nieuwenhuys B.E. Catalysis by Gold Nanoparticles. Gold Bull., 35(2), P. 39-45 (2002).

[13] Santra A.K., Goodman D.W.Oxide-supported metal clusters: models for heterogeneous catalysts. J. Phys.: Condens. Matter, 14, P. R31-R62 (2002).

[14] Hutchings G.J. Gold catalysis in chemical processing. Catal. Today, 72, P. 11-17 (2002).

[15] Haruta M. When Gold Is Not Noble: Catalysis by Nanoparticle. Chem. Record., 3(2), P. 75-87 (2003).

[16] Haruta M. Nanoparticulate Gold Catalysts for Low-Temperature CO Oxidation. J. New Mater. Electrochem. Syst., 7, P. 163-172 (2004).

[17] Meyer R., Lemire C., Shaikhutdinov S.K., Freund H.J. Surface chemistry of catalysis by gold. Gold Bull., 37, P. 72-124 (2004).

[18] Hutchings G.J. Catalysis by gold. Catal. Today, 100, P. 55-61 (2005).

[19] Haruta M., Tsubota S., Kobayashi T., Kageyama H., Genet M.J., Delmon B. Low-temperature oxidation of $\mathrm{CO}$ over gold supported on $\mathrm{TiO}_{2}$, alpha- $\mathrm{Fe}_{2} \mathrm{O}_{3}$ and $\mathrm{Co}_{3} \mathrm{O}_{4}$. J. Catal., 144, P. $175-192$ (1993).

[20] Tsubota S., Cunningham D.A.H., Bando Y., Haruta M. Prep. Catal. VI, Scientific Basis for the Preparation of Heterogeneous Catalysts. Elsevier, Amsterdam, 277 p. (1995).

[21] Boccuzzi F., Chiorino A., Tsubota S., Hurata M. FTIR Study of Carbon Monoxide Oxidation and Scrambling at Room Temperature over Gold Supported on $\mathrm{ZnO}$ and $\mathrm{TiO}_{2}$. J. Phys. Chem., 100(9), P. 3625-3631 (1996).

[22] Bamwenda G.R., Tsubota S., Nakamura T., Haruta M. The influence of the preparation methods on the catalytic activity of platinum and gold supported on $\mathrm{TiO}_{2}$ for $\mathrm{CO}$ oxidation. Catal. Lett., 44(1-2), P. 83-87 (1997).

[23] Valden M., Lai X., Goodman D.W. Onset of catalytic activity of gold clusters on titania with the appearance of nonmetallic properties. Science, 281, P. 1647-1650 (1998).

[24] Kozlov A.I., Kozlova A.P., Asakura K., Matsui Y., Kogure T., Shido T., Iwasawa Y. Supported gold catalysts prepared from a gold phosphine precursor and As-precipitated metal-hydroxide precursors: Effect of preparation conditions onthe catalytic performance. J. Catal., 196, P. 56-65 (2000).

[25] Claus P., Bruckner A., Mohr C., Hofmeister H. Supported gold nanoparticles from quantum dot to mesoscopic size scale: Effect of electronic and structural properties on catalytic hydrogenation of conjugated functional groups. J. Am. Chem. Soc., 122, P. 11430-11439 (2000).

[26] Schumacher B., Plzak V., Kinne K., Behm R.J. Highly active Au/ $\mathrm{TiO}_{2}$ catalysts for low-temperature CO oxidation: Preparation, conditioning and stability. Catal. Lett., 89(1-2), P. 109-114 (2003).

[27] Zanella R., Giorgio S., Shin C.-H., Henry C.R., Louis C. Characterization and reactivity in CO oxidation of gold nanoparticles supported on $\mathrm{TiO}_{2}$ prepared by deposition-precipitation with $\mathrm{NaOH}$ and urea. J. Catal., 222, P. 357-367 (2004).

[28] Schwartz V., Mullins D.R., Yan W., Chen B., Dai S., Overbury S.H. XAS study of Au supported on $\mathrm{TiO}_{2}$ : Influence of oxidation state and particle size on catalytic activity. J. Phys. Chem. B., 108(40), P. 15782-15790 (2004).

[29] Remediakis I.N., Lopez N., Nørskov J.K. CO oxidation on rutile-supported Au nanoparticles. Angew. Chem. Int. Ed., 44(12), P. 1824-1826 (2005).

[30] Bond C.G., Thompson D.T. Gold-catalysed oxidation of carbon monoxide. Gold Bull., 33, P. 41-50 (2000).

[31] Costello C.K., Yang J.H., Law H.Y., Wang Y., Lin J.N., Marks L.D., Kung M.D., Kung H.H. On the potential role of hydroxyl groups in $\mathrm{CO}$ oxidation over $\mathrm{Au} / \mathrm{Al}_{2} \mathrm{O}_{3}$. Appl. Catal. A, 243, P. 15-24 (2003).

[32] Sanchez A., Abbet S., Heiz U., Schneider W.D., Häkkinen H., Barnett R.N., Landman U. When gold is not noble: nanoscale gold catalysts. J. Phys. Chem.A, 103, P. 9573-9578 (1999).

[33] Yoon B., Häkkinen H., Landman U., Wörz A.S., Antonietti J.-M., Abbet S.,Judai K., Heiz U. Charging effects on bonding and catalyzed oxidation of $\mathrm{CO}$ on Au8 clusters on MgO. Science, 307, P. 403-407 (2005).

[34] Fu Q., Saltsburg H., Flytzani-Stephanopoulos M. Active Nonmetallic Au and Pt Species on ceria-based water-gas shift catalysts. Science, 301, P. 935-938 (2003). 
[35] Guzman J., Gates B.C. Structure and Reactivity of a Mononuclear Gold-Complex Catalyst Supported on Magnesium Oxide. Angew. Chem. Int. Ed., 42, P. 690-693 (2003).

[36] Molina L.M., Hammer B. Active role of oxide support during CO oxidation at Au/MgO. Phys. Rev. Lett., 90, P. 206102 (2003).

[37] Liu Z.-P., Gong X.-Q., Kohanoff J., Sanchez C., Hu P. Catalytic role of metal oxides in gold-based catalysts: a first principles study of $\mathrm{CO}$ oxidation on $\mathrm{TiO}_{2}$ supported Au. Phys. Rev. Lett., 91, P. 266102 (2003).

[38] Cleveland C.L., Landman U., Schaaff T.G., Shafigullin M.N., Stephens P.W., Whetten R.L. Structural evolution of smaller gold nanocrystals: The truncated decahedral motif. Phys. Rev. Lett., 79, P. 18731876 (1997).

[39] Uppenbrink J., Wales D.J. Structure and Energetics of Model Metal Clusters. J. Chem. Phys., 96, P. 8520-8534 (1992).

[40] Yacaman M.J., Fuentes S., Dominguez J.M. The effect of shape and crystal structure of small particles on their catalytic activity. Surf. Sci., 106, P. 472-477 (1981).

[41] Moraweck B., Renouprez A.J. EXAFS determination of the structure of small platinum particles. Surf. Sci., 106, P. 35-44 (1981).

[42] Berry C.R. Electron diffraction from small crystals. Phys. Rev., 88(3), P. 596-599 (1952).

[43] Wasserman H.J., Vermaak J.S. On the determination of a lattice contraction in very small silver particles. Surf. Sci., 22, P. 164-172 (1970).

[44] Purdum H., Montano P.A., Shenoy G.K., Morrison T.I. Extended-x-ray-absorption-fine-structure study of small Fe molecules isolated in solid neon. Phys. Rev.B, 25(7), P. 4412-4417 (1982).

[45] Montano P.A., Schulze W., Tesche B., Shenoy G.K., Morrison T.I. Extended x-ray-absorption finestructure study of Ag particles isolated in solid argon. Phys. Rev.B, 30, P. 672-677 (1984).

[46] Montano P.A., Purdum H., Shenoy G.K., Morrison T.I. X-ray absorption fine structure study of small metal clusters isolated in rare-gas solids. Surf. Sci., 156, P. 228-233 (1985).

[47] Heinemann K., Poppa H. In-situ TEM evidence of lattice expansion of very small supported palladium particles. Surf. Sci., 156, P. 265-274 (1985).

[48] Balerna A., Bernieri E., Picozzi P., Reale A., Santucci S., Burattini E.,Mobilio S. A structural investigation on small gold clusters by EXAFS. Surf. Sci., 156, P. 206-213 (1985).

[49] Balerna A., Bernieri E., Picozzi P., Reale A., Santucci S., Burattini E., Mobilio S., Extended x-rayabsorption fine-structure and near-edge-structure studies on evaporated small clusters of Au. Phys. Rev.B, 31(8), P. 5058-5065 (1985).

[50] Montano P.A., Shenoy G.K., Alp E.E., Schulze W., Urban J. Structure of copper microclusters isolated in solid argon. Phys. Rev.Lett.B., 56, P. 2076-2079 (1986).

[51] Pinto A., Pennisi A.R., Faraci G., D’Agostino G., Mobilio S., Boscherini F. Evidence for truncated octahedral structures in supported gold clusters. Phys. Rev.B, 51(8), P. 5315-5321 (1995).

[52] Klimenkov M., Nepijko S., Kuhlenbeck H., Bäumer M., Schlögl R., Freund H.-J. The structure of Pt-aggregates on a supported thin aluminum oxide film in comparison with unsupported alumina: a transmission electron microscopy study. Surf. Sci., 391, P. 27-36 (1997).

[53] Oudenhuijzen M.K., Bitter J.H., Koningsberger D.C. The Nature of the Pt-H bonding for strongly and weakly bonded hydrogen on platinum. A XAFS spectroscopy study of the $\mathrm{Pt}-\mathrm{H}$ antibonding shaperesonance and Pt-H EXAFS. J. Phys. Chem.B., 105(20), P. 4616-4622 (2001).

[54] Montano P.A., Zhao J., Ramanathan M., Shenoy G.K., Chulze W. EXAFS study of Ag, Fe and Ge microclusters. Physica B, 158, 1989(1-3), P. 242-242.

[55] Apai G., Hamilton J.F., Stöhr J., Thompson A. Extended X-ray-absorption fine structure of small $\mathrm{Cu}$ and Ni clusters: binding-energy and bond-length changes with cluster size. Phys. Rev. Lett., 43(2), P. 165-169 (1979).

[56] Crescenzi M., Picozzi P., Santucci S., Battistoni C., Mattogno G. Cluster growth of Cu on graphite: XPS, Auger and electron energy loss studies. Solid State Commun., 51(10), P. 811-815 (1984).

[57] Dupree R., Forwood C.T., Smith M.J.A. Conduction electron spin resonance in small particles of gold. Phys. Status Solidi, 24, P. 525-530 (1967).

[58] Monot R., Câtelain A., Borel J.P. Conduction electron spin resonance in small particles of pure gold. Phys. Lett. A., 34, P. 57-58 (1971).

[59] Mason M.G., Gerenser L.J., Lee S.T. Electronic structure of catalytic metal clusters studied by X-Ray photoemission spectroscopy. Phys. Rev. Lett., 39(5), P. 288-291 (1977). 
[60] Schmeisser D., Jocobi K., Kolb D.M. Photoemission study of matrix isolated $\mathrm{Cu}$ atoms and clusters. J. Chem. Phys., 75(11), P. 5300-5004 (1981).

[61] Lee S.-T., Apai G., Mason M.G., Benbow R., Hurych Z. Evolution of band structure in gold clusters as studied by photoemission. Phys. Rev. B., 23(2), P. 505-508 (1981).

[62] Kreibig U., Genzel L. Optical absorption of small metallic particles. Surf. Sci., 156, P. 678-700 (1985).

[63] Schmid G. Large clusters and colloids: Metals in the embryonic state. Chem. Rev., 92, P. 1709-1727 (1992).

[64] Binns C. Nanoclusters deposited on surfaces. Surf. Sci. Rep., 44, P. 1-49 (2001).

[65] Tamm I.E. Collection of scientific works. (In Russian). Nauka, Moscow, V. 1, 440 p. (1975).

[66] Morrison S.R. The Chemical Physics of Surfaces. Plenum Press, New York-London, 415 p. (1977).

[67] Davison S.G., Levine J.D. Surface States. Academic press, New York, 149 p. (1970).

[68] Flugge. S. Practical Quantum Mechanics. Springer, Berlin, Part I. 628 p. (1971).

[69] Efros Al.L., Efros A.L. Interband absorption of light in a semiconductor ball. Fizika i tekhnika poluprovodnikov, 16(7), P. 1209-1214 (1982). (In Russian).

[70] Kronig R.L, Penney W.G. Quantum mechanics of electrons in crystal lattices. Proc. Roy. Soc., 130, P. 499-513 (1931).

[71] Eyring H., Walter J., Kimball G.E. Quantum Chemistry. Wiley, New York, 345 p. (1944).

[72] Eyring H., Lin S.H., Lin S.M. Basic Chemical Kinetics. Wiley, New York, 493 p. (1980).

[73] W.Haberditzl. Structure of Matter and Chemical Bonding. Mir, oscow, 296 p. (1974). (in Russian).

[74] Moshfegh A.Z. Nanoparticle catalysts. J.Phys. D:Appl.Phys., 42(23), P. 233001 (2009).

[75] Miller J.T., Krops A.J., Zha Y., Regalluto J.R., L.Delannoy, C.Louis, Bus E., Bokhoven J.A. The effect of gold particle size on $\mathrm{Au}-\mathrm{Au}$ bond length and toward oxygen in supported catalysts. J.Catal., 240, P. 222-234 (2006). 